\title{
Investigation of the dose distribution for a cone beam CT system dedicated to breast imaging
}

\author{
Nico Lanconelli ${ }^{\text {a,b,* }}$, Giovanni Mettivier ${ }^{c, d}$, Sergio Lo Meo ${ }^{\text {a,b }}$, \\ Paolo Russo ${ }^{c, d}$
}

\author{
a Dipartimento di Fisica, Alma Mater Studiorum, Universita' di Bologna, Viale Berti-Pichat 6/2, \\ 40127 Bologna, Italy \\ b INFN, Sezione di Bologna, Viale Berti-Pichat 6/2, 40127 Bologna, Italy \\ ' Università di Napoli Federico II, Dipartimento di Scienze Fisiche, Via Cinthia, 80126 Napoli, Italy \\ dINFN, Sezione di Napoli, Via Cinthia, 80126 Napoli, Italy
}

Received 31 December 2011; received in revised form 21 April 2012; accepted 9 June 2012

Available online 2 July 2012

\author{
KEYWORDS \\ Cone-beam CT; \\ Dosimetry; \\ Monte Carlo; \\ Breast imaging PACS: \\ 87.59-e; \\ 87.53. Bn; \\ 87.57.Q-; \\ 87.59.E; \\ 87.57.rh
}

\begin{abstract}
Cone-beam breast Computed Tomography (bCT) is an X-ray imaging technique for breast cancer diagnosis, in principle capable of delivering a much more homogeneous dose spatial pattern to the breast volume than conventional mammography, at dose levels comparable to two-view mammography. We present an investigation of the three-dimensional dose distribution for a cone-beam CT system dedicated to breast imaging. We employed Monte Carlo simulations for estimating the dose deposited within a breast phantom having a hemiellipsoidal shape placed on a cylinder of $3.5 \mathrm{~cm}$ thickness that simulates the chest wall. This phantom represents a pendulant breast in a bCT exam with the average diameter at chest wall, assumed to correspond to a 5-cm-thick compressed breast in mammography. The phantom is irradiated in a circular orbit with an X-ray cone beam selected from four different techniques: $50,60,70$, and $80 \mathrm{kVp}$ from a tube with tungsten anode, $1.8 \mathrm{~mm}$ Al inherent filtration and additional filtration of $0.2 \mathrm{~mm} \mathrm{Cu}$. Using the Monte Carlo code GEANT4 we simulated a system similar to the experimental apparatus available in our lab. Simulations were performed at a constant free-inair air kerma at the isocenter $(1 \mu \mathrm{Gy})$; the corresponding total number of photon histories per scan was 288 million at $80 \mathrm{kVp}$. We found that the more energetic beams provide a more uniform dose distribution than at low energy: the $50 \mathrm{kVp}$ beam presents a frequency distribution of absorbed dose values with a coefficient of variation almost double than that for the $80 \mathrm{kVp}$ beam. This is confirmed by the analysis of the relative dose profiles along the radial (i.e. parallel to the "chest wall") and longitudinal (i.e. from "chest wall" to "nipple")
\end{abstract}

\footnotetext{
* Corresponding author. Dipartimento di Fisica, Alma Mater Studiorum, Universita' di Bologna, Viale Berti-Pichat 6/2, 40127 Bologna, Italy.
} E-mail address: nico.lanconelli@unibo.it (N. Lanconelli). 
directions. Maximum radial deviations are on the order of $25 \%$ for the $80 \mathrm{kVp}$ beam, whereas for the $50 \mathrm{kVp}$ beam variations around $43 \%$ were observed, with the lowest dose values being found along the central longitudinal axis of the phantom.

(c) 2012 Associazione Italiana di Fisica Medica. Published by Elsevier Ltd. All rights reserved.

\section{Introduction}

Conventional X-ray mammography (both in traditional and digital form) is still the most commonly used technique for breast cancer screening. However, the visualization and detection of cancer can be impaired in the acquired planar images because of the overlapping of normal tissue, soft tissue masses and calcifications. Computed Tomography (CT) can solve this problem by providing three-dimensional (3D) views of the breast anatomy. Cone-beam breast Computed Tomography (bCT) is an alternative method which usually employs a flat panel detector and a wide $X$ ray beam for generating $3 D$ images of the breast. On the contrary to traditional CT, here only the breast is exposed to the radiation beam [1]. This allows both to improve the image quality and to spare the rest of the patient's body from unnecessary radiation exposure. Furthermore, conebeam $C T$ is able to provide true 3D breast images with isotropic resolution and radiation dose comparable to twoview mammography $[2,3]$.

Dose delivery to tissues in conventional mammography is highly heterogeneous spatially [1]; indeed, most of the radiation is absorbed by surface tissue layers near to the entrance of the X-ray beam, whereas the opposite side will receive the lowest dose. From the analysis of dose histograms, Boone et al. [1] estimate that for two-view mammography of a $4.5-\mathrm{cm}$-thick compressed breast at a total Mean Glandular Dose (MGD) of $4 \mathrm{mGy}$, about $5 \%$ of the breast received an average of $14.2 \mathrm{mGy}$ and that $20 \%$ of the breast received an MGD greater than $10 \mathrm{mGy}$. On the other hand, $\mathrm{bCT}$ is capable of delivering a much more homogeneous dose pattern to the breast, especially at high kilovoltages $[1,4]$. This is mainly due to two factors: first the breast is irradiated from a plurality of positions, and second in bCT energies higher than those used in traditional mammography are usually employed. Thus, the homogeneity of the distribution of the dose should be considered a parameter of interest in the assessment of the overall quality of a bCT system [5-8].

Monte Carlo (MC) simulation is an important tool for dose assessment in cone-beam CT examinations $[9,10]$. MC simulations have been used for investigating the radiation dosimetry of CT systems dedicated to breast imaging $[1,2,4-6]$. GEANT4 is emerging as one of the most widespread MC simulation programs, even for applications in the medical field [11]. Indeed, GEANT4 was already used for estimating the dose deposited in soft tissues and for investigating the features of CT prototypes [12-14].

In order to better understand the dose distribution in a cone-beam CT system dedicated to breast imaging, in this paper we make use of MC simulations performed with GEANT4. We made simulations of the same experimental prototype developed by our group $[15,16]$. We estimated the dose deposited within a Polymethyl Methacrylate (PMMA) breast phantom irradiated with four different beams from an X-ray tube. The different energies of the four beams are expected to provide a different spatial distribution of absorbed dose in tissue, considering the difference in tissue attenuation coefficients at those energies. We report on the results of the dose distribution at various kilovoltages and a comparison with experimental measurements and published data, using PMMA phantoms simulating the average-size uncompressed during a bCT exam. Our main interest is to illustrate the differences in the relative dose distribution, not to give absolute dosimetric values.

\section{Materials and methods}

\section{Experimental prototype}

In this paper we simulate a cone-beam bCT prototype assembled in our laboratory at Federico II University in Naples. It consists of a modular, bench-top system with a step motor rotating gantry, a minifocus Tungsten anode Xray tube $(0.05 \mathrm{~mm}$ nominal focal spot size), a flat panel detector, custom acquisition software and commercial cone-beam CT reconstruction software. The X-ray tube has a continuous output, fixed tungsten anode, operated in the range $50-80 \mathrm{kVp}$ (1\% ripple). The tube presents an inherent filtration of $1.8 \mathrm{~mm} \mathrm{Al}$ and an additional filtration of $0.2 \mathrm{~mm}$ Cu was considered. This filtration was chosen because it has already been demonstrated that it is capable of providing a more uniform distribution of dose inside the phantom, in comparison to thinner $\mathrm{Cu}$ filtrations [7]. The scanner is mounted on an optical bench and housed in a shielded cabinet. The mechanical assembly of the scanner contains two rotating arms positioned along the horizontal axis of the tomograph, which hold the X-ray tube and the detector, respectively. The prototype is equipped with 8 computerdriven step motors for controlling rotations and translations necessary for achieving the tomographic acquisition with selectable image magnification and acquisition field-ofview. The system is comprised of a CMOS Flat Panel Detector with a $0.15-\mathrm{mm}$-thick Csl scintillator layer and a sensitive area of $12 \mathrm{~cm} \times 12 \mathrm{~cm}$ with $50 \mu \mathrm{m} \times 50 \mu \mathrm{m}$ pixels. A detailed description of the experimental apparatus can be found elsewhere [7,15-17]. In this study we do not simulate the X-ray imaging detector, since we focus our attention on the dose distribution within the breast.

\section{Breast phantom}

We estimated via $M C$ simulations the absorbed dose in a homogeneous phantom made of PMMA $\left(1.19 \mathrm{~g} / \mathrm{cm}^{3}\right)$ with a shape which approximates a pendulant average breast in a bCT exam. A sketch of the simulated phantom is shown in Fig. 1a. We assumed that the uncompressed breast presents a $14-\mathrm{cm}$ diameter at the chest wall, which is the average "effective" breast diameter at chest wall reported by 

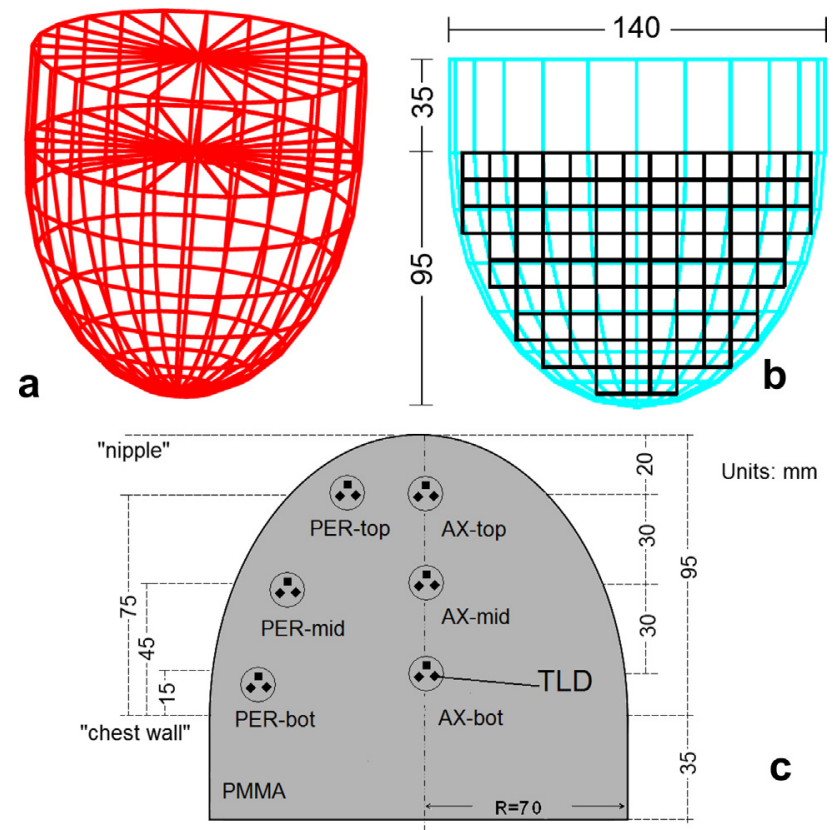

Figure 1 a) Sketch of the simulated hemiellipsoidal phantom (in red). b) The dose is registered within cubical voxels located within the phantom. The cylindrical base simulates the chest wall. The phantom diameter at its base $(14 \mathrm{~cm})$ corresponds to the average effective diameter of the pendulant breast in a bCT exam. c) Scheme of a real PMMA phantom showing the positions of the TLDs at midplane in the phantom, indicating the axial ( $\mathrm{AX}$ ) and peripheral (PER) positions close to the chest wall (bot), in the middle (mid) and close to the nipple (top) of the breast phantom. At each position three TLD-100 chips were placed. (For interpretation of the references to color in this figure legend, the reader is referred to the web version of this article.)

Boone et al. [3], with a length of the pendulant breast of 1.36 times its radius (close to the value of 1.5 times the radius assumed by Boone et al.). Thus, the breast phantom was modeled as a hemiellipsoid of rotation of half-axes of $7 \mathrm{~cm}$ (breast radius at chest wall) and $9.5 \mathrm{~cm}$ (breast length), with a cylindrical base $3.5 \mathrm{~cm}$ thick for simulating the chest wall. The phantom was placed at the scanner isocenter, with its longitudinal axis (chest wall to nipple) at a distance $R_{1}$ of $385 \mathrm{~mm}$ from the focal spot of the X-ray tube. The distance $R_{2}$ of the detector from the isocenter was $120 \mathrm{~mm}$ so that system magnification was $\left[\left(R_{1}+R_{2}\right) /\right.$ $\left.R_{1}\right]=1.31$. A grid of cubical voxels was simulated within the phantom, in order to register the dose in different locations, as shown in Fig. 1b. Each voxel has a $1-\mathrm{cm}$ side and for covering the breast entirely 697 voxels were considered. The hemiellipsoidal phantom volume (without its cylindrical base) is $975 \mathrm{~cm}^{3}$ so that this mesh covers over $71 \%$ of the simulated breast volume. For each simulation, the total energy deposited in each voxel was scored (in $\mathrm{MeV}$ ), and finally converted into average dose values expressed in Gy, given the voxel mass. For a correct estimate of the dose, the grid of voxels considered must be completely inscribed within the breast phantom. Considering the shape of the phantom and the size of the voxels, it turns out that is not possible to cover the whole breast. If one would consider smaller voxels, the coverage would increase. But in that case even the simulation time would increase noticeably, for getting the same statistical error. However, we believe that our choice is a good tradeoff between simulation time and coverage of the breast: most of the breast is covered, except for some peripheral regions.

For the $80 \mathrm{kVp}$ beam we also considered two additional simulated conditions. First, a second type of tissue more similar to the real one was used: breast tissue $(50 / 50)$ composed of $50 \%$ fibroglandular tissue and $50 \%$ fat with density $0.985 \mathrm{~g} / \mathrm{cm}^{3}$. In addition, a second breast phantom size was simulated: a hemiellipsoid smaller than the first one, with half-axes of $6 \mathrm{~cm}$ (radius at chest wall) and $9 \mathrm{~cm}$ (breast length). Indeed, unpublished observations made in a population of 200 women undergoing mammography screening at Naples Cancer Institute show that the average breast size is about $12 \mathrm{~cm}$, slightly lower than the average value reported for a population of 200 women in California $(14 \mathrm{~cm})$ [3].

\section{X-ray beams}

The X-ray tube spectra have been generated using SpekCalc, an executable code freely available on the web for calculating the $X$-ray emission spectra from tungsten anodes tubes [18]. The spectra have been generated for four different tube voltages $(50,60,70$, and $80 \mathrm{kVp})$ at $1 \mathrm{keV}$ interval for an inherent filtration of $1.8 \mathrm{~mm} \mathrm{Al}$ with additional filtration of $0.2 \mathrm{~mm} \mathrm{Cu}$. Figure 2 shows the spectra calculated for the four beams considered in this paper. Some characteristics of these spectral models are listed in Table 1.

\section{Simulated system}

MC simulations were run by using GEANT4, a generalpurpose toolkit which can simulate the transport of several particle types in a wide range of energies [11]. Originally developed for simulating high-energy physics experiments, it is also used in various areas of application, including

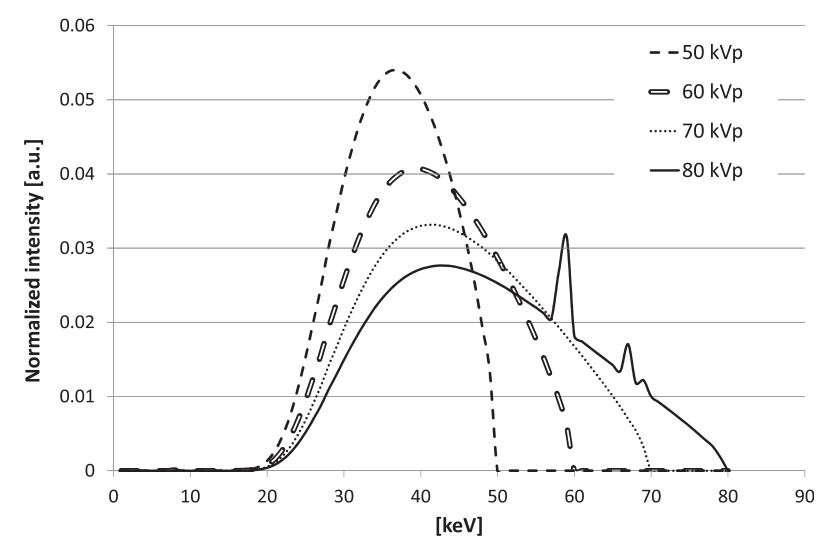

Figure 2 Normalized X-ray spectra for W anode tube derived from the SpekCalc software [18]. For all the beams we considered an inherent filtration of $1.8 \mathrm{~mm} \mathrm{Al}$ and an added filtration of $0.2 \mathrm{~mm} \mathrm{Cu}$. 
Table 1 Parameters of the four spectra considered in this paper.

\begin{tabular}{llllll}
\hline & $\begin{array}{l}\text { Half value layer } \\
{[\mathrm{mmAl}]}\end{array}$ & $\begin{array}{l}\text { Average energy } \\
{[\mathrm{keV}]}\end{array}$ & $\begin{array}{l}\text { Effective energy } \\
\text { (Al) }[\mathrm{keV}]\end{array}$ & $\begin{array}{l}\text { Tube output } \\
\text { at isocenter } \\
{[\mathrm{mGy} / \mathrm{mAs}]}\end{array}$ & $\begin{array}{l}\text { Photon fluence per } \\
\text { mGy air kerma } \\
{\left[\mathrm{photons} / \mathrm{cm}^{2}\right]}\end{array}$ \\
\hline $50 \mathrm{kVp}$ & 3.22 & 37.6 & 34.5 & 0.038 & $2.79 \times 10^{8}$ \\
$60 \mathrm{kVp}$ & 3.92 & 42.2 & 37.5 & 0.072 & $3.24 \times 10^{8}$ \\
$70 \mathrm{kVp}$ & 4.53 & 46.3 & 40.0 & 0.113 & $3.56 \times 10^{8}$ \\
$80 \mathrm{kVp}$ & 5.15 & 50.3 & 42.6 & 0.165 & $3.80 \times 10^{8}$ \\
\hline
\end{tabular}

medical physics. With GEANT4 a broad range of physics effects can be simulated, such as hadronic, electromagnetic, and optical processes. With the latest releases of GEANT4 the electromagnetic interactions can be simulated with very good accuracy down to energies smaller than $1 \mathrm{keV}$. Users can also select various physics' lists (e.g. Standard, Penelope, Low-energy), through which different electromagnetic processes can be considered; each list implements different models of radiation transport and makes use of different cross-section databases for the various effects [19]. Simulations presented in this paper have been performed with the Standard Electro-Magnetic (EM) package, comprising the following effects: Photoelectric Effect, Compton Scattering and Gamma Conversion for the transport of photons and Multiple Scattering, Ionization, and Bremsstrahlung for the electrons. The standard EM model is considered valid in a range of energy that fits well with our case (from $1 \mathrm{keV}$ to about $10 \mathrm{PeV}$ ). In a previous work [13] we evaluated this package with the low energy libraries available with GEANT4 in a similar problem (transport of photons and electrons in a homogeneous medium in a range of energy compatible with that considered in this paper). No substantial differences between the two outcomes were observed, whereas the simulation time increased considerably with the Penelope model. As a consequence, we decided to use the standard model in this research.

In order to estimate the absorbed dose within the breast phantom, we simulated a tomographic acquisition in a circular orbit with 360 projections over $360^{\circ}$, with the phantom longitudinal axis at the scanner isocenter $(385 \mathrm{~mm}$ from the focal spot). Simulations were performed by varying the X-ray tube kivoltage $(50,60,70$, and $80 \mathrm{kVp})$ at a constant air kerma at isocenter $(1 \mu \mathrm{Gy})$ for the four considered photon spectra and by keeping an additional filtration of $0.2 \mathrm{~mm}$ Cu for all the beams. The focal spot was simulated as a point source and the cone-beam angle was $30^{\circ}$, sufficient for irradiating the entire phantom. In reality, the focal spot of the X-ray tube is not a point $(50 \mu \mathrm{m}$ nominal size). The number of photons simulated for each beam condition was selected as that necessary for giving the same free-in-air air kerma at the isocenter $(1 \mu \mathrm{Gy})$ independently of tube voltage. The total deposited energy in PMMA was scored for each and every voxel positioned within the breast phantom. The simulated air kerma at isocenter was much less than typically used in a bCT exam (1 $\mu \mathrm{Gy}$ vs. $6.4 \mathrm{mGy}$ at $80 \mathrm{kVp}$ for a $14-\mathrm{cm}$-thick uncompressed breast of $50 \%$ glandular fraction [3]). However, this allowed to reduce computation times while performing simulations with as many as 288 million total number of photon histories per scan at $80 \mathrm{kVp}$.
The uncertainty on the dose registered for each voxel was estimated by using a history by history method [20]. In practice, for each history we stored both the energy and the energy squared value deposited in each voxel. At the end of the simulation we accumulate the registered values and we thus estimate the uncertainty of the scored dose. The uncertainty depends on the number of simulated photons; it turns out that the errors on the registered dose were always smaller than $1 \%$. We are aware that we are simulating a number of photons much lower than those considered in the experimental measurements. However, we believe that this represents a good tradeoff between the precision in our outcomes and the simulation time required.

\section{Experimental validation of the Monte Carlo}

The simulation outcomes are validated with experimental data from our prototype and reported in a previous paper [7]. To this end we compared the distribution of dose obtained for the 14-cm diameter hemiellipsoidal phantom made of PMMA, irradiated with either of the four considered X-ray beams. The experimental dose value was measured by means of six termoluminescent dosimeters (TLDs) placed in different positions within the phantom (Fig. 1). Table 2 shows a comparison with the experimental measurements acquired with our prototype in the same conditions and on the same hemiellipsoidal phantom [7]. In that paper the dose was measured in six different positions within the phantom: three on the longitudinal axis (position named $A X$ ) and three on peripheral positions (named PER). In both cases the TLD chips were positioned at three different heights: at about $15 \mathrm{~mm}$ from the chest wall

Table 2 Percent difference between the dose data presented in this paper and that reported in [7], for each kilovoltage and position in the phantom. The comparison has been achieved for the five positions of the TLDs considered in that paper (i.e. PER-bot, AX-mid, PER-mid, AX-top, and PER-top. For the detailed description of the location of these positions, see Fig. 1). Data are normalized with respect to the innermost position (i.e. AX-bot position defined in [7]).

\begin{tabular}{llcccc}
\hline & PER bot & AX mid & PER mid & AX top & PER top \\
\hline $50 \mathrm{kVp}$ & $-7 \%$ & $-3 \%$ & $-10 \%$ & $-9 \%$ & $-10 \%$ \\
$60 \mathrm{kVp}$ & $-6 \%$ & $3 \%$ & $-2 \%$ & $0 \%$ & $-3 \%$ \\
$70 \mathrm{kVp}$ & $-5 \%$ & $-2 \%$ & $-6 \%$ & $-6 \%$ & $-8 \%$ \\
$80 \mathrm{kVp}$ & $-5 \%$ & $3 \%$ & $-1 \%$ & $-1 \%$ & $-3 \%$ \\
\hline
\end{tabular}


(bot), at about $45 \mathrm{~mm}$ from the chest wall (mid), and close to the nipple (at about $75 \mathrm{~mm}$ from the chest wall - top position). Data were normalized to the dose registered in the innermost position (i.e. AX-bot). Considering that the overall dose uncertainties in [7] were on the order of $5 \%$, agreement between simulations and experimental data presented in Table 2 are good for all the beams, except for the $50 \mathrm{kVp}$ beam, where there is an overestimation of the dose on the order of several percent for all the considered positions. We point out that there are some factors which are not considered in present simulations, including the heel effect, the presence of the mechanical gantry of the system and of the $\mathrm{Pb}$ shielding, which may contribute to a slightly different distribution of the dose within the phantom (due to the scattered radiation) and which can cause some discrepancy between simulated and experimental data, expected to be of the same sign and amount as the deviations observed here. As a further validation of our MC simulation, we calculated the ratio of dose in PMMA ( $\mu \mathrm{G} y)$ to free-in-air air kerma ( $\mu \mathrm{Gy}$ ) for our hemiellipsoidal phantom of $14 \mathrm{~cm}$ diameter, at varying kilovoltages; these values have been compared with the air dose ratio measured in [2] for 10, 13, 16 and 20-cm-diameter PMMA cylindrical phantoms (Fig. 3). The air dose ratio was defined as the air kerma in air at the center of the PMMA phantom, divided by the air kerma free-in-air at the same axial position, without the phantom; measurements in [2] were performed with an ionization chamber placed in an air-filled cavity at the center of the phantom. Figure 3 shows a deviation less than $10 \%$ of our simulation data (open symbols) from the interpolated value at $14-\mathrm{cm}$-diameter between the data reported by Boone et al. [2] (closed symbols). The agreement is considered fairly good.

\section{Results and discussion}

Figure 4 shows a 3D scatter plot of the dose distribution obtained for the 697 voxels in the PMMA phantom irradiated with the $80 \mathrm{kVp}$ beam. Data are normalized to the minimum

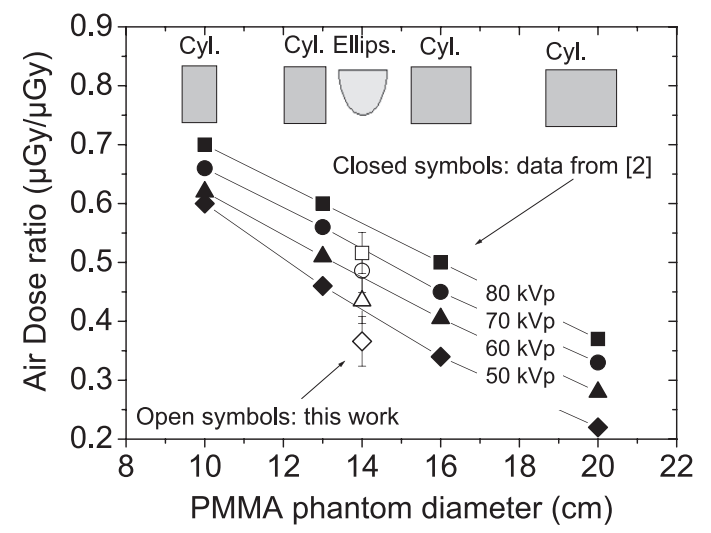

Figure 3 The ratio of dose in PMMA to free-in-air air kerma at the hemiellipsoidal phantom axis, as evaluated in the present work (open symbols) at $50-80 \mathrm{kVp}$, is plotted with comparison to measured air dose ratios reported in [2] at the same kilovoltages for cylindrical PMMA phantoms of $10-20 \mathrm{~cm}$ diameter and $15.2 \mathrm{~cm}$ height.

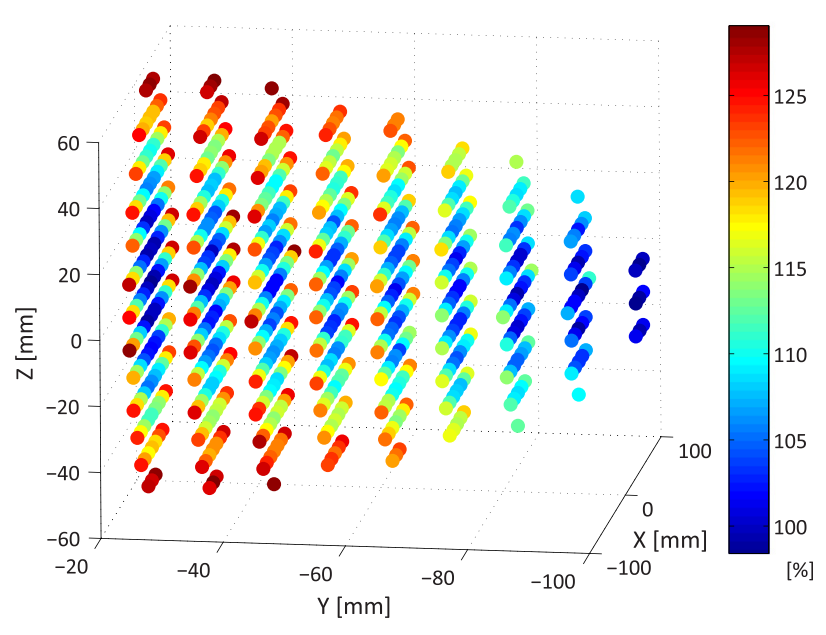

Figure $43 \mathrm{D}$ dose distribution of the PMMA breast phantom irradiated with the $80 \mathrm{kVp}$ beam. The 3D position $(x, y, z)$ of the different voxels is represented along the 3 coordinate axes, whereas their dose values are shown with a color map. Data are normalized to the minimum value registered. The $y$-axis is directed along the scanner rotation axis, with chest wall at left and nipple at right.

value registered, located near the chest wall and on the central axis of the phantom, for all four dose distributions from $50 \mathrm{kVp}$ to $80 \mathrm{kVp}$. Voxels which received the highest dose are those located at the periphery of the phantom (near the skin), as expected. It is worth noting that the spread of the frequency distribution of dose is very limited, since the highest dose is about $25 \%$ higher than the lowest one. We also notice that, thanks to the tomographic geometry, there is a quasi isotropic dose distribution around the central axis of the breast (i.e. longitudinal $-y$ direction in Fig. 4). The distribution of the dose for the other three beams follows the same pattern, even though the range of the deposited doses is wider at low energies.

Figure 5 shows 2D-plots of the dose absorbed in four axial planes inside the 14-cm diameter phantom, both at 50 and $80 \mathrm{kVp}$. Similar data at 60 and $70 \mathrm{kVp}$ are omitted for conciseness. The planes have been selected at an axial position with a distance of $15,35,55$, and $85 \mathrm{~mm}$ from the chest wall, for each kilovoltage. Dose values ranging from $0.28 \mu \mathrm{Gy}$ to $0.46 \mu \mathrm{Gy}$ (at $50 \mathrm{kVp}$ ), and from $0.44 \mu \mathrm{Gy}$ to $0.60 \mu \mathrm{Gy}$ (at $80 \mathrm{kVp}$ ) are observed; in each $2 \mathrm{D}$ plot at any given axial position $y$, the dose decreases from the periphery toward the center of the phantom. This decreasing trend is evident especially near the chest wall. These radial differences are much more limited than those observed in conventional mammography, where variations in the distributions of glandular dose as large as $600 \%$ have been reported and $20 \%$ of the $5-\mathrm{cm}$ thick compressed breast received more than double the average glandular dose $[1,4]$. The variations in registered dose are more moderate along the longitudinal direction, from the chest to the nipple (i.e. along the $y$ direction). Again, the other investigated beams follow the same trend, but with more accentuated variations between the inner and the peripheral regions.

The histogram of the distributions of dose values registered in the grid of cubical voxels for two of the considered 

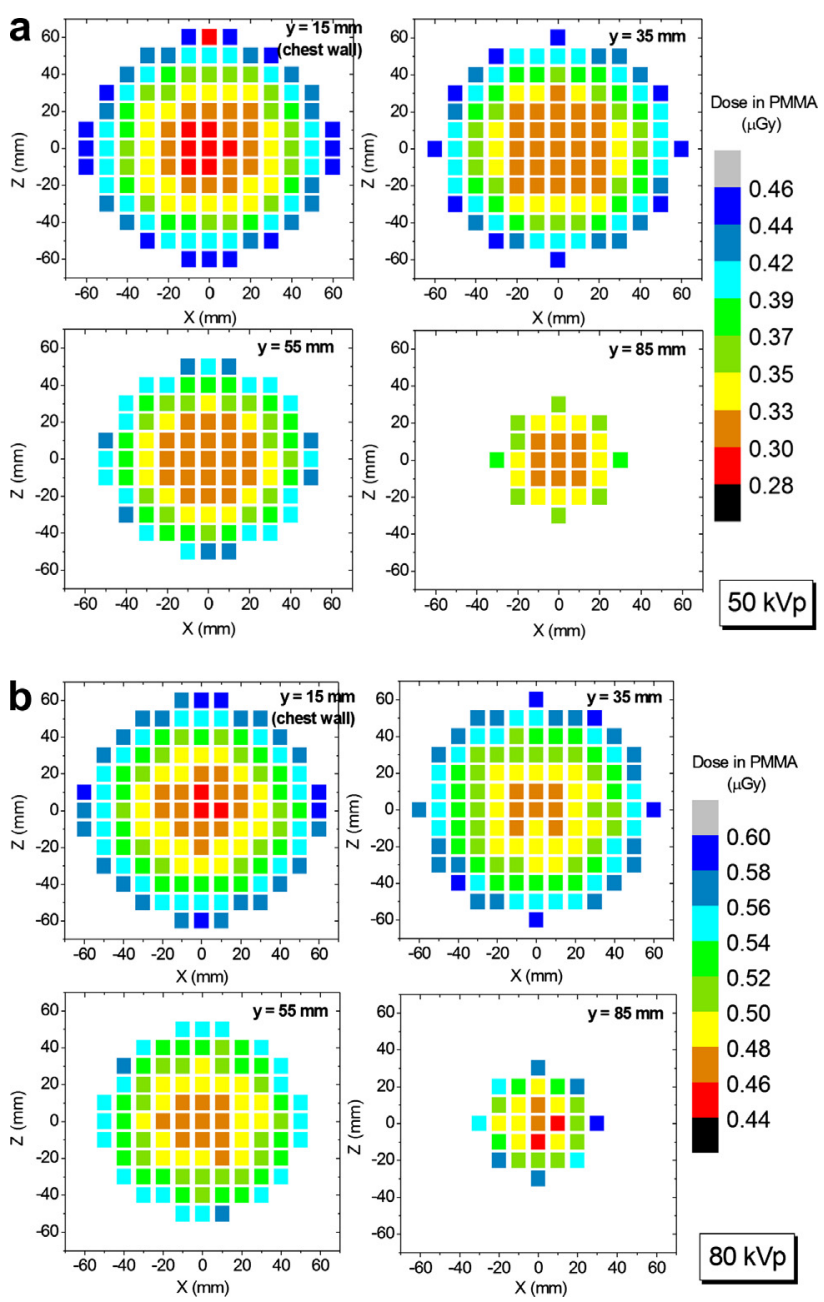

Figure $52 \mathrm{D}$ plot of the distribution of the absorbed dose in the 14-cm PMMA phantom, evaluated in a slice $(10-\mathrm{mm}$-thickness) located at a distance of $(15,35,55$, and $85 \mathrm{~mm})$ from the chest wall. Each square symbol represents the simulated dose in a $10 \mathrm{~mm} \times 10 \mathrm{~mm}$ voxel.

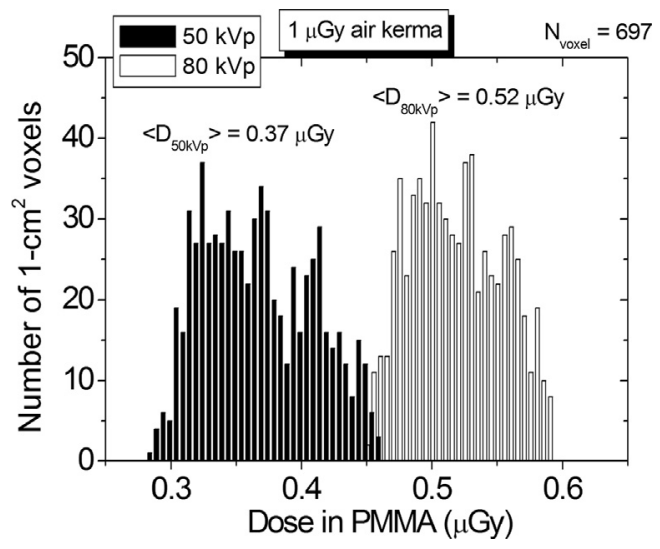

Figure 6 Histogram of the dose in PMMA breast phantom for two different beams: $50 \mathrm{kVp}$ (black), and $80 \mathrm{kVp}$ (white). The free-in-air air kerma at isocenter was fixed at $1 \mu \mathrm{Gy}$. beams ( $50 \mathrm{kVp}$ and $80 \mathrm{kVp}$ ) is reported in Fig. 6, where dose histograms are shown in order to compare the distributions of dose values achieved in the breast phantom with the different beams.

In this figure we can note that the dose deposition by the most energetic beam is higher on average, with respect to the low-energy beam. This is due to the more penetrating characteristic of the high-energy beams, which deposit energy at greater depths in the phantom, as already reported by other authors [2]. The two intermediate beams are not presented in this plot for the sake of clarity, but their histograms are positioned intermediately, as expected. Second, we would remark that the $50 \mathrm{kVp}$ histogram is noticeably wider than the $80 \mathrm{kVp}$ one. This confirms the fact that more energetic beams provide more homogeneous dose distributions (i.e. narrower dose histograms). We also note that, the histogram of the $50 \mathrm{kVp}$ beam presents a pronounced tail on the right (toward high doses). This indicates that the use of low-energy beams would deliver high dose to a significant number of voxels (those located at small depths). Three quantitative parameters for measuring the uniformity of the dose deposition are summarized in Table 3 for the four beams. We considered the following figures of merit: the difference between the maximum and minimum values of the histogram, the skewness of the distribution (i.e. the ratio of the third central moment of the distribution and the standard deviation raised to the third power), and the Coefficient of Variation (CoV), defined as the ratio between the standard deviation and the mean of the distribution. The first parameter is a measure of the extension of the dose distribution, the second one indicates its asymmetry and the third one gives an estimate of its uniformity. We can see that the distribution becomes increasingly more homogeneous and with a less pronounced tail as the beam kilovoltages increases, as demonstrated by both the difference between maximum and minimum and by the skewness at varying kilovoltages. As a consequence, the uniformity of the dose deposition also increases with energy, since the $50 \mathrm{kVp}$ beam presents a CoV almost double than the $80 \mathrm{kVp}$ one.

The (normalized) mean and standard deviation of the distribution of dose values inside the PMMA phantom as a function of the kilovoltage are depicted in Fig. 7. This figure shows the increase of the average value of the absorbed dose in the $14-\mathrm{cm}$ phantom at increasing kilovoltages from 50 to $80 \mathrm{kVp}$ (normalized to the value recorded at $50 \mathrm{kVp}$ ). The trend is similar for both our data (open symbols) and for the corresponding normalized (at $50 \mathrm{kVp}$ )

Table 3 Comparison of dose uniformity for the four considered beams in terms of difference between the maximum and minimum dose registered, skewness of the dose distribution, and Coefficient of Variation (CoV), defined as the ratio between the standard deviation and the mean of the distribution.

\begin{tabular}{lllr}
\hline & Max-Min $[\mu G y]$ & Skewness & CoV \\
\hline $50 \mathrm{kVp}$ & 0.172 & 0.26 & $11.5 \%$ \\
$60 \mathrm{kVp}$ & 0.166 & 0.23 & $8.9 \%$ \\
$70 \mathrm{kVp}$ & 0.158 & 0.21 & $7.6 \%$ \\
$80 \mathrm{kVp}$ & 0.139 & 0.16 & $6.7 \%$ \\
\hline
\end{tabular}




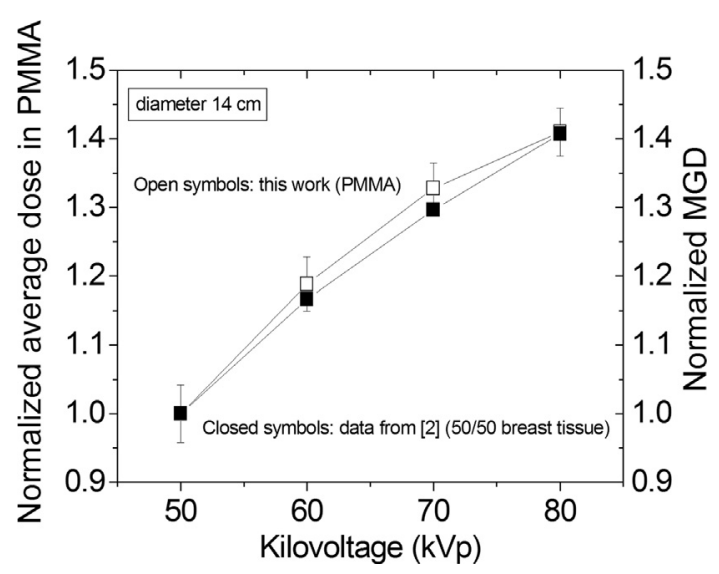

Figure 7 Simulated absorbed dose in PMMA hemiellipsoidal phantom (mean \pm std. dev., normalized to the value at $50 \mathrm{kVp}$ ) for varying kilovoltages (open symbols). Also shown for comparison are data of normalized MGD calculated for a 14$\mathrm{cm}$-diameter 50/50 breast according to [2] (closed symbols).

data derived from [2] (closed symbols) (see Fig. 15 in that paper) for a $14-\mathrm{cm}$ breast diameter of $50 \%$ glandular fraction. The agreement is quite satisfactory as regards the trend of the average dose increase from 50 to $80 \mathrm{kVp}$.

In Fig. 8 is shown the radial profile of the dose distribution in the PMMA phantom for the four investigated beams. Profiles were estimated along horizontal planes in the phantom at three different distances from the chest wall: $15 \mathrm{~mm}, 45 \mathrm{~mm}$, and $75 \mathrm{~mm}$ (same distances from the chest wall as in 5). Here, the position at $0 \mathrm{~mm}$ shown on the abscissa of the plots corresponds to the voxels located on the central axis of the phantom (longitudinal axis). Data for each beam were normalized to the minimum value registered for that beam. The profiles for the $50-k V p$ and $60-k V p$ beams present more marked variations, with respect to the 70- and $80-\mathrm{kVp}$ beams, radially from the inner part of the breast toward the skin. This trend can be noticed both near the chest wall (Fig. 8, plot a), and also in the proximity of the nipple (Fig. 8, plot c). For the 50-kVp beam we observed a variation up to $50 \%$ in peripheral voxels, with respect to the minimum dose registered in voxels on the central axis close to the chest wall. The beam at 70 and $80 \mathrm{kVp}$ shows very similar trends, indicating that the choice of high-energy beams is really capable of delivering a more homogeneous dose in homogeneous tissues.

Figure 9 shows the longitudinal profiles of the dose distribution for the four beams. Also in this case the two less energetic beams show the more accentuated variations (dose values at the nipple up to around 15\% higher than those registered at the chest wall). On the contrary, the 70$\mathrm{kVp}$ and $80-\mathrm{kVp}$ beams give a more uniform longitudinal distribution of the dose delivery, the fluctuations being confined within a few percent.

In order to provide a quantitative comparison among the various beams, we considered two parameters $(\Delta=$ edgecenter/center and $\delta=$ nipple - chest_wall/chest_wall), which measure the relative dose variation along the profiles on the radial and longitudinal directions shown in Figs. 8 and 9. The edge position was at the PER-bot location, the center (chest wall) position was at the AX-bot and the nipple
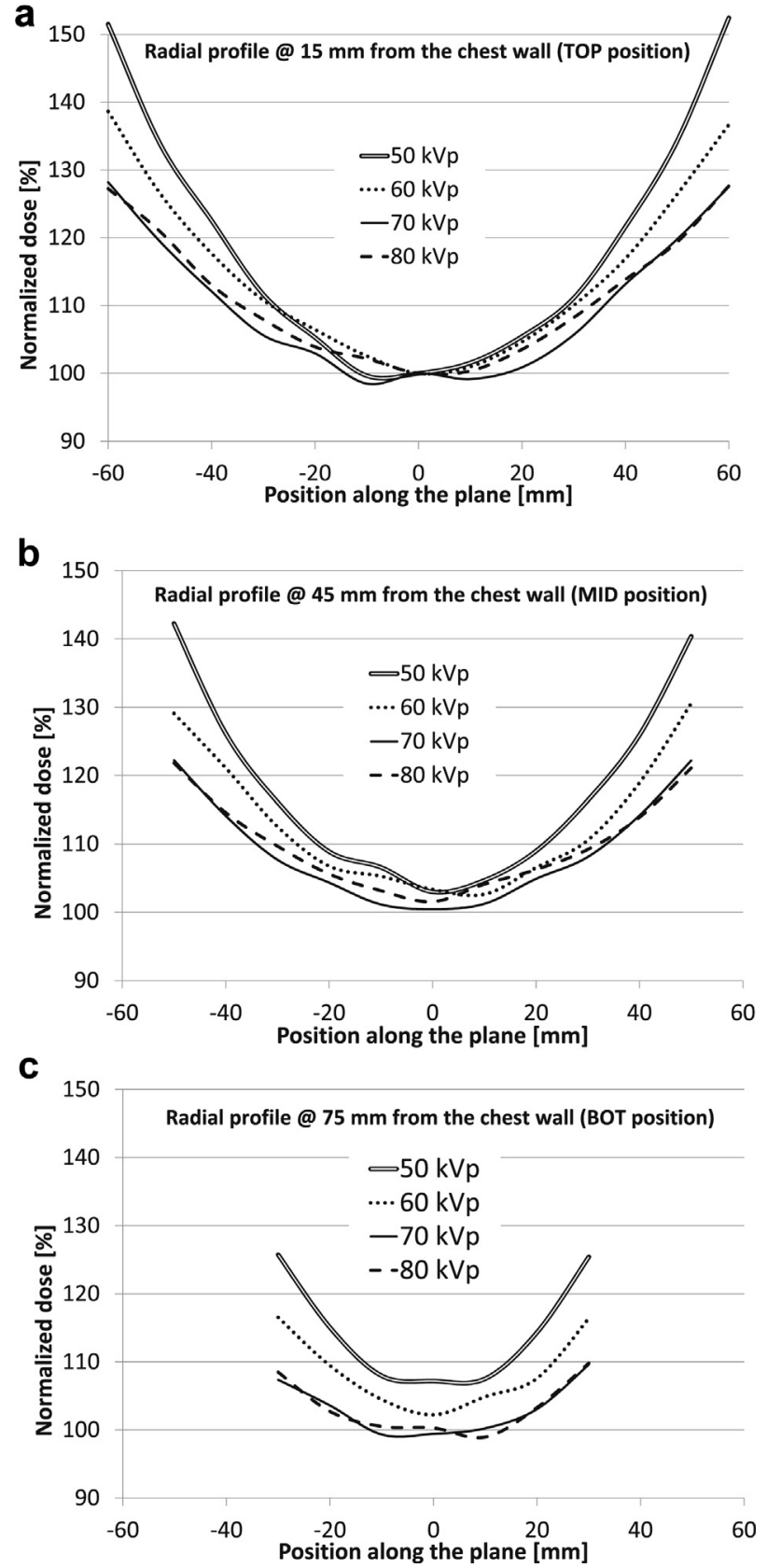

Figure 8 Radial profiles of the dose distribution in the PMMA phantom for the four investigated beams at three different distances from the chest wall: $15 \mathrm{~mm}$ (a), $45 \mathrm{~mm}$ (b), and $75 \mathrm{~mm}$ (c). Data are normalized to the minimum value at the central position registered for the four beams.

position was at the AX-top location in the phantom (Fig. 1c). Table 4 summarizes the results calculated in the PMMA phantom for the four beams. As already noted, the two most energetic beams show more limited variations, both in the radial and in the longitudinal direction, with respect to $50--60-k V p$ beams. In particular for the $80 \mathrm{kVp}$ beam, relative dose variations as measured by the $\Delta$ metric reach a maximum of about $25 \%$ in the radial direction and are confined within $2 \%$ on the longitudinal profile. On the other hand, for the $50 \mathrm{kVp}$ beam variations up to $43 \%$ and about $10 \%$ are observed in the radial and longitudinal 


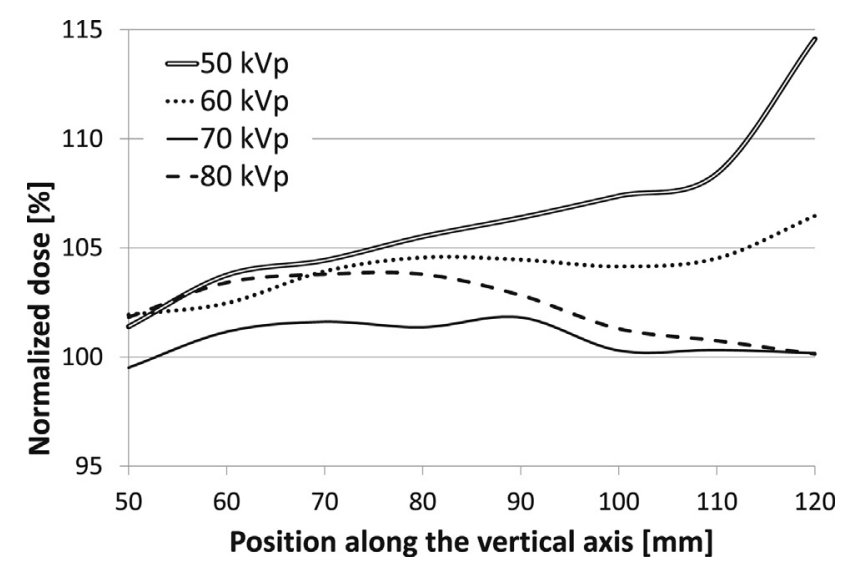

Figure 9 Longitudinal profiles (i.e. along the central axis of the PMMA phantom $-y$ axis in Fig. 4) of the dose distribution for the four investigated beams. Data are normalized to the minimum value registered for the four beams.

direction, respectively. Thacker and Glick reported a similar analysis achieved with simulated monochromatic $X$-ray beams [5]. In their paper they found a decreasing $\Delta$ as the energy increases, and for $40 \mathrm{keV}$ and $60 \mathrm{keV}$ photons, they estimated $\Delta$ values of about $40 \%$ and $14 \%$, respectively, as also reported in [8]. Considering that the average energy of our polychromatic beams is about $38 \mathrm{keV}$ and $50 \mathrm{keV}$ for the $50 \mathrm{kVp}$ and $80 \mathrm{kVp}$, respectively, the agreement with the data reported in [5] is satisfactory. Similar results ( $\Delta$ around $25 \%$ for $80 \mathrm{kVp}$ beam) were also shown for a cylindric paraffin phantom in [21]. Crotty et al. in a recent paper measured similar values $(\Delta=29 \%$ and $\mathrm{CoV}$ of around 9\%) using a similar hemiellipsoidal phantom than the one considered in this paper, but a different X-ray beam (tungsten $60 \mathrm{kVp}$ with additional Ce filter) [22]. In addition, previous experimental measurements achieved with our prototype reported in [7] showed $\Delta=18 \%$ and $\delta=-2 \%$ with the same $80 \mathrm{kVp}$ beam considered here, in good agreement with the simulated data presented in this paper. In a subsequent paper similar results ( $\Delta$ up to $25 \%$ ) were presented for a very similar prototype [8]. In that case the values measured for the $\delta$ parameter were different from those obtained in this paper, possibly due to the different longitudinal position of the focal spot with respect to the one considered here $(\delta$ being strongly dependent on the position of the focal spot projection on the longitudinal axis).

Figure 10 shows the radial profiles of the dose distribution achieved in the $12-\mathrm{cm}$ diameter phantom at $80 \mathrm{kVp}$ for the

Table 4 Variation of the dose values on radial $(\Delta)$ and longitudinal $(\delta)$ profiles, respectively (see text for a complete description). Radial profiles have been estimated on a plane at a distance of $15 \mathrm{~mm}$ from the chest wall.

\begin{tabular}{llr}
\hline & $\Delta$ & \multicolumn{1}{c}{$\delta$} \\
\hline $50 \mathrm{kVp}$ & $43 \%$ & $11 \%$ \\
$60 \mathrm{kVp}$ & $33 \%$ & $4 \%$ \\
$70 \mathrm{kVp}$ & $24 \%$ & $0 \%$ \\
$80 \mathrm{kVp}$ & $22 \%$ & $-2 \%$ \\
\hline
\end{tabular}

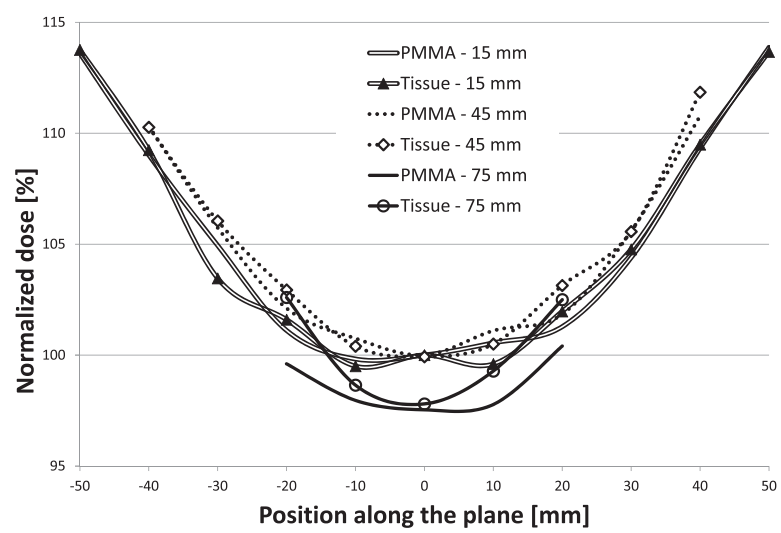

Figure 10 Radial profiles of the dose distribution in the $12 \mathrm{~cm}$ phantom for the $80 \mathrm{kVp}$ beams for two tissues (PMMA and breast tissue) at three different distances from the chest wall: $15 \mathrm{~mm}, 45 \mathrm{~mm}$, and $75 \mathrm{~mm}$. Data are normalized to the minimum value at the central position registered.

two investigated tissues (PMMA and 50/50 breast tissue). Figure 10 shows that the different materials produce similar distributions as already observed by Crotty et al. for antropomorphic breast phantoms [22]. However, in the $14-\mathrm{cm}$ phantom the average dose absorbed in breast tissue is different from that in PMMA (variation of 6\%), and the histogram of dose values is slightly broader for PMMA (CoV of $6.7 \%$ for PMMA, and $5.8 \%$ for breast tissue). This is related to the observation that denser media present dose distributions broader than less dense materials, as noted by Crotty et al. for water and for oil [22]. These differences are greatly reduced in the $12-\mathrm{cm}$ phantom, where both average dose and CoV are not significantly different for the two tissues.

Apart from the issue of dose distribution, it is worth remarking some considerations about the image quality that $\mathrm{bCT}$ is able to provide. Some groups have already reported that dedicated cone-beam CT systems are able to give equal or superior performance, with respect to conventional mammography, in terms of visualization of breast lesions with doses comparable to those delivered in mammography [1,23-25]. In general, the detectability of a lesion is determined by the combined effect of spatial resolution and contrast resolution capability of the clinical unit. Although bCT presents lower spatial resolution than mammography (in our experimental setup, the resolution was about $0.6 \mathrm{~mm}$ FWHM in the 14-cm PMMA phantom), its significant higher contrast resolution allows the detection of microcalcifications and masses. This has been demonstrated both with breast phantoms and with clinical examinations [24,25]. In particular, clinical investigations showed that most calcifications and all the masses detected with mammography are also detected with cone-beam bCT [24]. The good performance in terms of image quality is also confirmed when high energies ( $80 \mathrm{kVp}$ or higher) are employed $[1,23]$. In this case, clinical studies on 65 patients demonstrated that the overall CT performance was equal to mammography for visualization of breast lesions. In particular, bCT was significantly better than mammography for visualization of masses, whilst mammography outperformed bCT for microcalcifications [23]. 


\section{Conclusion}

In this paper we presented an investigation of the dose distribution for a cone-beam CT system dedicated to breast imaging. We used MC simulations for estimating the dose deposited within a breast phantom. The phantom was irradiated with either of four different beams: $50,60,70$, and $80 \mathrm{kVp}$ with tungsten anode and additional filtration of $0.2 \mathrm{~mm} \mathrm{Cu}$. It turned out that the more energetic beams are capable of providing a much more uniform dose distribution: the $50 \mathrm{kVp}$ beam presents a CoV almost double than the $80 \mathrm{kVp}$ one. This is also confirmed by the relative dose variation along the profiles on the radial and longitudinal directions. In particular, radial fluctuations reach a maximum difference of about $25 \%$ for the $80 \mathrm{kVp}$ beam, whereas are about $43 \%$ for the $50 \mathrm{kVp}$ beam. The simulated data presented here illustrate a thorough investigation of the dose distribution and are in good agreement with experimental measurements achieved with the prototype available in our lab and with data coming from the literature, using breast phantoms.

\section{References}

[1] Boone JM, Nelson TR, Lindfors KK, Seibert JA. Dedicated breast CT: radiation dose and image quality evaluation. Radiology 2001;221(3):657-67.

[2] Boone JM, Shah N, Nelson TR. A comprehensive analysis of $\mathrm{DgN}_{C T}$ coefficients for pendant-geometry cone-beam breast computed tomography. Medical Physics 2004;31(2):226-35.

[3] Boone JM, Kwan ALC, Seibert JA, Shah N, Lindfors KK, Nelson TR. Technique factors and their relationship to radiation dose in pendant geometry breast CT. Medical Physics 2005;32(12):3767-76.

[4] Sechopoulos I, Feng SSJ, D’Orsi CJ. Dosimetric characterization of a dedicated breast computed tomography clinical prototype. Medical Physics 2010;37(8):4110-20.

[5] Thacker SC, Glick SJ. Normalized glandular dose (DgN) coefficients for flat-panel CT breast imaging. Physics in Medicine and Biology 2004;49(24):5433-44.

[6] Yi Y, Lai CJ, Han T, Zhong Y, Shen Y, Liu X, et al. Radiation doses in cone-beam breast computed tomography: a Monte Carlo simulation study. Medical Physics 2011;38(2):589-97.

[7] Russo P, Lauria A, Mettivier G, Montesi M, Villani N. Dose distribution in cone-beam breast computed tomography: an experimental phantom study. IEEE Transactions on Nuclear Science 2010;57(1):366-74.

[8] Russo P, Coppola T, Mettivier G. Distribution of absorbed dose in cone-beam breast computed tomography: a phantom study with radiochromic films. IEEE Transactions on Nuclear Science 2010;57(4):2220-9.

[9] Spezi E, Downes P, Radu E, Jarvis R. Monte Carlo simulation of an $x$-ray volume imaging cone beam CT unit. Medical Physics 2009;36(1):127-36.

[10] Morant J, Salvadó M, Casanovas R, Hernández-Girón I, Velasco E, Calzado A. Validation of a Monte Carlo simulation for dose assessment in dental cone beam CT examinations. Physica Medica 2012;28(3):200-9.
[11] Agostinelli S, Allison J, Amako K, Apostolakis J, Araujo H, Arce $P$, et al. Geant4-a simulation toolkit. Nuclear Instruments and Methods in Physics Research Section A: Accelerators, Spectrometers, Detectors and Associated Equipment 2003; 506(3):250-303.

[12] Zahra N, Frisson T, Grevillot L, Lautesse P, Sarrut D. Influence of Geant4 parameters on dose distribution and computation time for carbon ion therapy simulation. Physica Medica 2010; 26(4):202-8.

[13] Pacilio M, Lanconelli N, Lo Meo S, Betti M, Montani L, Aroche LAT, et al. Differences among Monte Carlo codes in the calculations of voxel $S$ values for radionuclide targeted therapy and analysis of their impact on absorbed dose evaluations. Medical Physics 2009;36(5):1543-52.

[14] Mettivier G, Russo P, Lanconelli N, Lo Meo S. Evaluation of scattering in cone-beam breast computed tomography: a Monte Carlo and experimental phantom study. IEEE Transactions on Nuclear Science 2010;57(5):2510-7.

[15] Russo P, Mettivier G, Lauria A, Montesi M. X-ray cone-beam breast computed tomography: phantom studies. IEEE Transactions on Nuclear Science 2010;57(1):160-72.

[16] Mettivier G, Russo P, Cesarelli M, Ospizio R, Passeggio G, Roscilli L, et al. Dedicated scanner for laboratory investigations on cone-beam CT/SPECT imaging of the breast. Nuclear Instruments and Methods in Physics Research Section A: Accelerators, Spectrometers, Detectors and Associated Equipment 2011;629(1):350-6.

[17] Mettivier G, Russo P. Measurement of the mtf of a cone-beam breast computed tomography laboratory scanner. IEEE Transactions on Nuclear Science 2011;58(3):703-13.

[18] Poludniowski G, Landry G, DeBlois F, Evans PM, Verhaegen F. Spekcalc: a program to calculate photon spectra from tungsten anode x-ray tubes. Physics in Medicine and Biology 2009; 54(19):N433-8.

[19] Poon E, Verhaegen F. Accuracy of the photon and electron physics in GEANT4 for radiotherapy applications. Medical Physics 2005;32(6):1696-711.

[20] Walters BRB, Kawrakow I, Rogers DWO. History by history statistical estimators in the [small-caps beam] code system. Medical Physics 2002;29(12):2745-52.

[21] Chen L, Shen Y, Lai CJ, Han T, Zhong Y, Ge S, et al. Dual resolution cone beam breast CT: a feasibility study. Medical Physics 2009;36(9):4007-14.

[22] Crotty DJ, Brady SL, Jackson DC, Toncheva GI, Anderson CE, Yoshizumi TT, et al. Evaluation of the absorbed dose to the breast using radiochromic film in a dedicated CT mammotomography system employing a quasi-monochromatic x-ray beam. Medical Physics 2011;38(6):3232-45.

[23] Lindfors KK, Boone JM, Nelson TR, Yang K, Kwan ALC, Miller DF. Dedicated breast CT: Initial clinical experience. Radiology 2008;246(3):725-33.

[24] O'Connell A, Conover DL, Zhang Y, Seifert P, Logan-Young W, Lin CFL, et al. Cone-beam CT for breast imaging: radiation dose, breast coverage, and image quality. American Journal of Roentgenology 2010;195(2):496-509.

[25] Ning R, Conover D, Yu Y, Zhang Y, Cai W, BetancourtBenitez R, et al. A novel cone beam breast CT scanner: system evaluation. In: Hsieh J, editor. . In: Flynn MJ, editor. Medical imaging 2007: physics of medical imaging, vol. 6510. SPIE; 2007. p. 651030-31-651030-39. 